\title{
Cavallo's Multiplier for in situ Generation of High Voltage
}

\author{
S. M. Clayton, ${ }^{a}$ T. M. Ito, ${ }^{a}$ J. C. Ramsey, ${ }^{a, 1}$ W. Wei, ${ }^{a, 2}$ M. A. Blatnik, ${ }^{b}$ B. W. Filippone, ${ }^{b}$ and G. \\ M. Seidel ${ }^{c}$ \\ ${ }^{a}$ Los Alamos National Laboratory, \\ Los Alamos, New Mexico 87545, U.S.A. \\ ${ }^{b}$ W. K. Kellogg Radiation Laboratory, California Institute of Technology, \\ Pasadena, California 91125, U.S.A. \\ ${ }^{c}$ Department of Physics, Brown University, \\ Providence, Rhode Island 02912, U.S.A. \\ E-mail: sclayton@lanl.gov
}

\begin{abstract}
Aвstract: A classic electrostatic induction machine, Cavallo's multiplier, is suggested for in situ production of very high voltage in cryogenic environments. The device is suitable for generating a large electrostatic field under conditions of very small load current. Operation of the Cavallo multiplier is analyzed, with quantitative description in terms of mutual capacitances between electrodes in the system. A demonstration apparatus was constructed, and measured voltages are compared to predictions based on measured capacitances in the system. The simplicity of the Cavallo multiplier makes it amenable to electrostatic analysis using finite element software, and electrode shapes can be optimized to take advantage of a high dielectric strength medium such as liquid helium. A design study is presented for a Cavallo multiplier in a large-scale, cryogenic experiment to measure the neutron electric dipole moment.
\end{abstract}

KEYwords: High voltage, cryogenic detectors

ArXIV EPrint: 1803.07665

\footnotetext{
${ }^{1}$ Present address: Oak Ridge National Laboratory, Oak Ridge, Tennessee 37831, U.S.A.

2Present address: W. K. Kellogg Radiation Laboratory, California Institute of Technology, Pasadena, California 91125, U.S.A.
} 


\section{Contents}

1 Introduction 1

2 Cavallo's multiplier 2

2.1 Principle of operation 2

2.2 Heat Generation 5

2.3 Parallel-Plate Capacitor Approximation 6

3 Demonstration apparatus $\quad 8$

4 Cavallo's multiplier in a neutron EDM experiment $\quad 11$

5 Discussion and Conclusion $\quad 13$

$\begin{array}{lll}6 & \text { Acknowledgements } & 14\end{array}$

\section{Introduction}

Many physics experiments require application of high voltage to create strong electrostatic fields. For example, electric dipole moment experiments seek energy level shifts when a particle is subject to a strong electric field. High voltages are also used in some particle detectors, such as time projection chambers (TPCs), which use an electric field to drift charged particles toward a detector grid. Often a very high voltage is required in an isolated or harsh environment, such as cryogenic, where a power supply cannot operate.

High voltage produced external to the apparatus requires a feedthrough. Cryogenic experiments, in particular those performed at sub-Kelvin temperatures, present the additional challenge that the heat load must be small. Feedthroughs are subject to leakage current, which can be a significant heat input to the central part of the apparatus. In addition, the high voltage conductors may need to be thermally anchored at heat shields. This presents an additional challenge, as it requires thermally conducting but electrically insulating material. Generating the very high voltage inside the central part of the apparatus, or multiplying a much more modest high voltage fed into the apparatus, avoids the problems with feedthroughs.

In this paper, we suggest reviving a classic high voltage multiplier machine, the Cavallo multiplier [1], to establish high voltage in cryogenic environments with greatly reduced demand on the voltage feedthrough. The high voltage generator of the Pelletron [2], which is based on electrostatic induction like the Cavallo multiplier and may be thought of as its modern descendant, is capable of higher performance and, in particular, continuous current delivery. However, the simplicity of the Cavallo multiplier in terms of mechanical actuation and electrostatic design may be an advantage in certain experiments, where only very low current is required. 


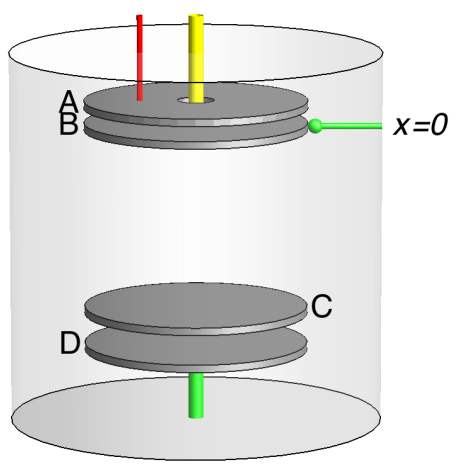

a) Induce charge onto $B$

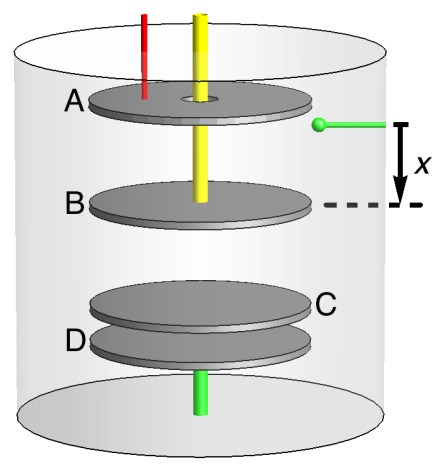

b) Move charge

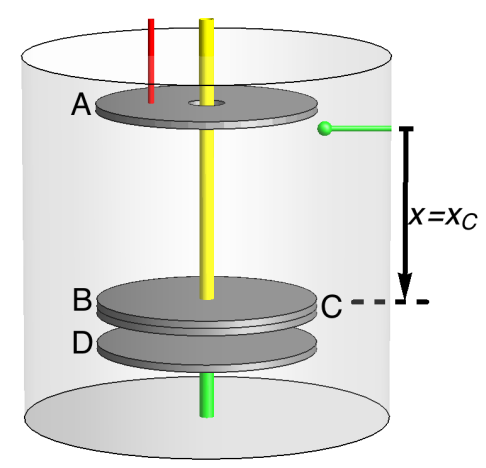

c) Transfer charge to $\mathrm{C}$

Figure 1. Conceptual drawing of a Cavallo multiplier, showing the steps to charge the capacitor formed by the isolated electrode (C) and ground including (grounded) plate D. An external power supply is connected to plate A with a wire (red line in drawing) to apply a constant voltage. The movable plate (B) is held with an insulating rod (yellow). a) Plate B is grounded with a pin (top right) when positioned near plate A, acquiring a charge due to its capacitance with A. b) Plate B is disconnected from ground, which traps the induced charge, and is moved toward plate C. c) Plate B contacts plate C, transferring charge according to capacitances of the system. The steps are repeated, accumulating charge on C.

The organization of this paper is as follows. First, we explain the principle of the Cavallo multiplier, discuss its limiting voltage gain, solve for the voltages on the electrodes during the charging process, and estimate heat produced by the generator. We then show results from a roomtemperature demonstration apparatus, comparing predicted to actual voltage on the high voltage electrode throughout the charging process. Finally, we present a design study for a Cavallo multiplier suitable for a large-scale, cryogenic apparatus to measure the neutron electric dipole moment.

\section{Cavallo's multiplier}

Cavallo's multiplier was invented in the late 18th century to amplify small voltages for detection with electroscopes [1]. The machine uses electrostatic induction and mechanical movement to accumulate charge onto a fixed capacitor, with a corresponding increase in voltage across the capacitor.

\subsection{Principle of operation}

A conceptual drawing of a Cavallo multiplier is shown in figure 1. Plates A, C, and D are fixed, while plate $\mathrm{B}$ is movable. Plate $\mathrm{A}$ is held at a modest high voltage through connection to an external power supply. The electrically insulated plate $\mathrm{C}$ and grounded plate $\mathrm{D}$ comprise the capacitor to be charged to high voltage by the apparatus. Plate $\mathrm{B}$ is initially positioned near plate $\mathrm{A}$, where it makes contact with a grounding pin, acquiring an electrical charge $Q_{B} \approx-C_{A B} V_{A}$ through electrostatic induction according to its mutual capacitance $C_{A B}$ with plate A (this expression for $Q_{B}$ is approximate due to parasitic capacitance with plate $\mathrm{C}$, which will be considered below). Plate $\mathrm{B}$ is moved away from $\mathrm{A}$, breaking contact with the ground pin and thus maintaining its initial charge 
$Q_{B}$. Plate B is then brought into contact with plate $\mathrm{C}$, transferring charge between the plates. When plates B and $\mathrm{C}$ are subsequently separated, the total charge $Q_{B}+Q_{C}$ will be distributed between plates $\mathrm{B}$ and $\mathrm{C}$ according to their relative mutual capacitances with other electrodes in the system, with the amount of charge left on plate $\mathrm{C}$

$$
Q_{C}^{\prime}=\frac{\left(Q_{B}+Q_{C}\right) C_{C G}}{C_{C G}+C_{B G}},
$$

in which only mutual capacitances with ground G (plate D plus the ground shell) are considered.

It may be useful to clarify the meaning of mutual capacitance $C_{i j}$ between two conductors $i$ and $j$. This is the constant of proportionality between the amount of additional charge $Q_{i j}$ on conductor $i$ and its voltage difference with plate $j, Q_{i j}=C_{i j}\left(V_{i}-V_{j}\right)$. The total charge on conductor $i$ is obtained by summing over all other conductors in the system, $Q_{i}=\sum_{j \neq i} C_{i j}\left(V_{i}-V_{j}\right)$. The total charge can also be expressed in terms of the electric field at its surface, $Q_{i}=\int_{S_{i}} \epsilon_{0} \vec{E} \cdot d \vec{a}$ (integral form of Gauss's Law), where the integral is over the surface of conductor $i$. In the Cavallo multiplier, there is no charge on the sides of plates $\mathrm{B}$ and $\mathrm{C}$ facing each other just after they make contact because the field is zero between the plates; we see that plate C "shields" B from plate D, leaving only the much smaller capacitance to the ground shell to contribute to $C_{B G}$.

Another cycle begins by moving B back to its position near A and in contact with the grounding pin. Starting with $Q_{C}=0$, after $n$ cycles the charge accumulated onto $\mathrm{C}$ is

$$
Q_{C n}=Q_{B} \frac{\epsilon\left(1-\epsilon^{n}\right)}{1-\epsilon},
$$

where $\epsilon \equiv C_{C G} /\left(C_{C G}+C_{B G}\right)$. The amount of charge loaded onto plate $\mathrm{C}$ in the limit of infinite number of cycles is

$$
\lim _{n \rightarrow \infty} Q_{C n}=Q_{B} \frac{C_{C G}}{C_{B G}},
$$

equivalent to the condition of no net charge transferred to $\mathrm{C}$ after contact with $\mathrm{B}\left(Q_{B}^{\prime}=Q_{B}\right)$. This amounts to a maximum voltage achievable on plate $\mathrm{C}$ of $V_{C}^{\max }=-V_{A} C_{A B} / C_{B G}$, independent of the capacitance $C_{C G}$.

As the total amount of charge on plate $\mathrm{C}$ increases, it may be important to account for its influence on the initial charge loaded onto plate B,

$$
Q_{B}^{a}=-C_{A B}^{a} V_{A}-C_{B C}^{a} V_{C}^{a},
$$

where the superscript $a$ indicates that parameters correspond to when B is in its initial position next to A. Similarly, we can account for the influence of plate A on the charge that remains stuck on B, when B is in contact with $\mathrm{C}$,

$$
Q_{B}^{c}=C_{A B}^{c}\left(V_{C}^{c}-V_{A}\right)+C_{B G}^{c} V_{C}^{c}
$$

where the superscript $c$ labels this position. The condition for maximum possible charge on $\mathrm{C}$ is when all of the charge loaded onto B remains stuck on the B electrode, $Q_{B}^{a}=Q_{B}^{c}$. Equivalently, the charge on plate $\mathrm{C}$ is unchanged, $Q_{C}^{c}=Q_{C}^{a}$, giving an additional expression,

$$
Q_{C}=C_{C G}^{c} V_{C}^{c}=\left(C_{C G}^{a}+C_{B C}^{a}\right) V_{C}^{a} .
$$


Combining eqs. 2.4-2.6 and $Q_{B}^{a}=Q_{B}^{c}$ gives an expression for the maximum voltage on $\mathrm{C}$ (when $\mathrm{B}$ remains in contact with $C$ ) relative to the voltage $V_{A}$ fed into the system,

$$
-\frac{V_{C}^{1, \max }}{V_{A}}=\frac{C_{A B}^{a}-C_{A B}^{c}}{C_{B G}^{c}+C_{A B}^{c}+\kappa C_{B C}^{a}} \equiv G^{\max },
$$

where $\kappa \equiv C_{C G}^{c} /\left(C_{C G}^{a}+C_{B C}^{a}\right)$ and we define the maximum voltage gain $G^{\max }$. Typically, $\kappa \approx 1$ and $G^{\max }$ does not depend on the load capacitance $C_{C G}$.

To discharge plate $\mathrm{C}, V_{A}$ is reduced, zeroed, or reversed depending on how much charge is to be removed in a given cycle. If $V_{A}=0, V_{C}$ approaches zero asymptotically with number of cycles. Fully discharging plate $\mathrm{C}$ requires setting $V_{A}$ to its slightly reversed value (same polarity as plate $\mathrm{C}$ ) to remove the last bit of charge.

The machine in figure 1 can be further understood by generalizing eqs. 2.5 and 2.6 to any plate B position $x$,

$$
\begin{aligned}
& Q_{B}=C_{A B}\left(V_{B}-V_{A}\right)+C_{B C}\left(V_{B}-V_{C}\right)+C_{B G} V_{B}, \\
& Q_{C}=C_{B C}\left(V_{C}-V_{B}\right)+C_{C G} V_{C}
\end{aligned}
$$

in which the mutual capacitances and voltages are functions of $x$. We neglect the mutual capacitance between the fixed plates $\mathrm{A}$ and C. The solutions for $V_{B}$ and $V_{C}$ with given $Q_{B}$ and $Q_{C}$ are

$$
\begin{aligned}
V_{B} & =\frac{Q_{B}+C_{A B} V_{A}+\eta Q_{C}}{C_{A B}+C_{B G}+\eta C_{C G}}, \\
V_{C} & =\frac{Q_{C}}{C_{C G}+C_{B C}}+\eta V_{B},
\end{aligned}
$$

with $\eta \equiv C_{B C} /\left(C_{C G}+C_{B C}\right)$.

As plate $B$ approaches plate $C$ to within an infinitesimal gap, we expect their mutual capacitance $C_{B C}$ to diverge to infinity. In this limit, as evident from eq. 2.11, plates B and C approach the same voltage prior to touching. The electric field between the plates during the approach depends on details of the geometry. If the plates are parallel planes of infinite extent, the field between them is

$$
\begin{aligned}
E_{B C}(x) & =\frac{V_{B}(x)-V_{C}(x)}{\Delta x} \\
& =\left((1-\eta(x)) V_{B}(x)-\frac{\eta(x) Q_{C}}{C_{B C}(x)}\right) / \Delta x \\
& =\frac{V_{B}(x) C_{C G}-Q_{C}}{\left(C_{C G}+C_{B C}(x)\right) \Delta x},
\end{aligned}
$$

where $\Delta x=x_{C}-x$ is the distance between plates B and $\mathrm{C}$. To calculate the field just before the plates touch, we take the limit of eq. 2.14 as $\Delta x \rightarrow 0$ and $C_{B C} \gg C_{C G}$,

$$
\lim _{x \rightarrow x_{C}} E_{B C}(x)=\lim _{x \rightarrow x_{C}} \frac{V_{B}(x) C_{C G}-Q_{C}}{C_{B C}(x) \Delta x} .
$$

Applying the same limit to eq. 2.10, we have

$$
\lim _{x \rightarrow x_{C}} V_{B}(x)=\frac{Q_{B}+Q_{C}+C_{A B} V_{A}}{C_{A B}+C_{B G}+C_{C G}}
$$


and the numerator in eq. 2.15 is finite as the plates approach. The electric field is finite just before contact if $C_{B C}$ diverges as $(\Delta x)^{-1}$ (or faster), a condition met by a parallel plate capacitor: $C_{B C}(\Delta x)=\epsilon A / \Delta x$, with plate area $A$ and dielectric constant between the plates $\epsilon$, giving

$$
\lim _{x \rightarrow x_{C}} E_{B C}(x)=\frac{V_{B}\left(x_{C}\right) C_{C G}-Q_{C}}{\epsilon A} .
$$

The actual field before contact between real electrodes depends on details of the surfaces, electrode shapes and their alignment. In general, we expect the field to diverge prior to contact and create a spark.

\subsection{Heat Generation}

The electrostatic potential energy of the charged system is provided by the mechanical energy required to move plate $\mathrm{B}$ between plates $\mathrm{A}$ and $\mathrm{C}$, plus the external power supply sourcing or sinking charge on plate A. Here, we consider heat generation in the Cavallo multiplier itself, not including work done by the external power supply. There are three possible sources of dissipation in this otherwise efficient process: sparking between electrodes, heat from charges flowing across conductor surfaces, and drag from moving plate B through a fluid medium (unless in vacuum).

Due to the high electric field as plate B approaches $\mathrm{C}$, at some point prior to contact charge will flow between the plates via spark or field emission and dissipate energy in the process. The maximum energy available to the spark is the difference in electrostatic configuration energy before and after the event, $W_{\text {spark }}=W_{\text {initial }}-W_{\text {final }}$, with the configuration energy given by

$$
W=\frac{1}{2} \sum_{i} Q_{i} V_{i}
$$

and the sum is over all electrodes in the system. This energy is the upper limit on heating due to sparks between the electrodes and will be estimated with a parallel plate model in Sec. 2.3. There can also be a spark as plate $\mathrm{B}$ returns to $x=0$ and contacts the grounding pin, though this can be suppressed by temporarily reducing $V_{A}$ to an appropriate setting depending on the residual charge $Q_{B}^{c}$ left on B, before B contacts the ground pin.

Another source of heat is due to rearrangement of charges on electrodes with non-zero surface resistivity $\rho_{S}$, for example from the top to bottom surfaces of plate B as it moves from initial proximity to plate $\mathrm{A}$ down to plate $\mathrm{C}$ :

$$
\begin{aligned}
W_{\mathrm{q}} & \sim \Delta t I^{2} \rho_{S} \\
& \sim \Delta t\left(Q_{B} / \Delta t\right)^{2} \rho_{S} \\
& \sim \rho_{S} Q_{B}^{2} / \Delta t,
\end{aligned}
$$

where $\Delta t$ is a characteristic time interval over which charge flows from the top to bottom of the plate. Similarly, as plate B approaches $\mathrm{C}$, charges are repelled from the side of $\mathrm{C}$ facing $\mathrm{B}$, leaving the surface facing $\mathrm{B}$ with charge approaching $-Q_{B}$ in the limit of $\mathrm{B}$ and $\mathrm{C}$ almost touching. The amount of heat generated can be estimated from eq. 2.19 with appropriate characteristic $\rho_{S}$ and $\Delta t$.

If the multiplier is immersed in a classical fluid, heat is generated by the drag force on the movable plate $\mathrm{B}$. If the flow about the plate is laminar, the drag force depends on the shape and 


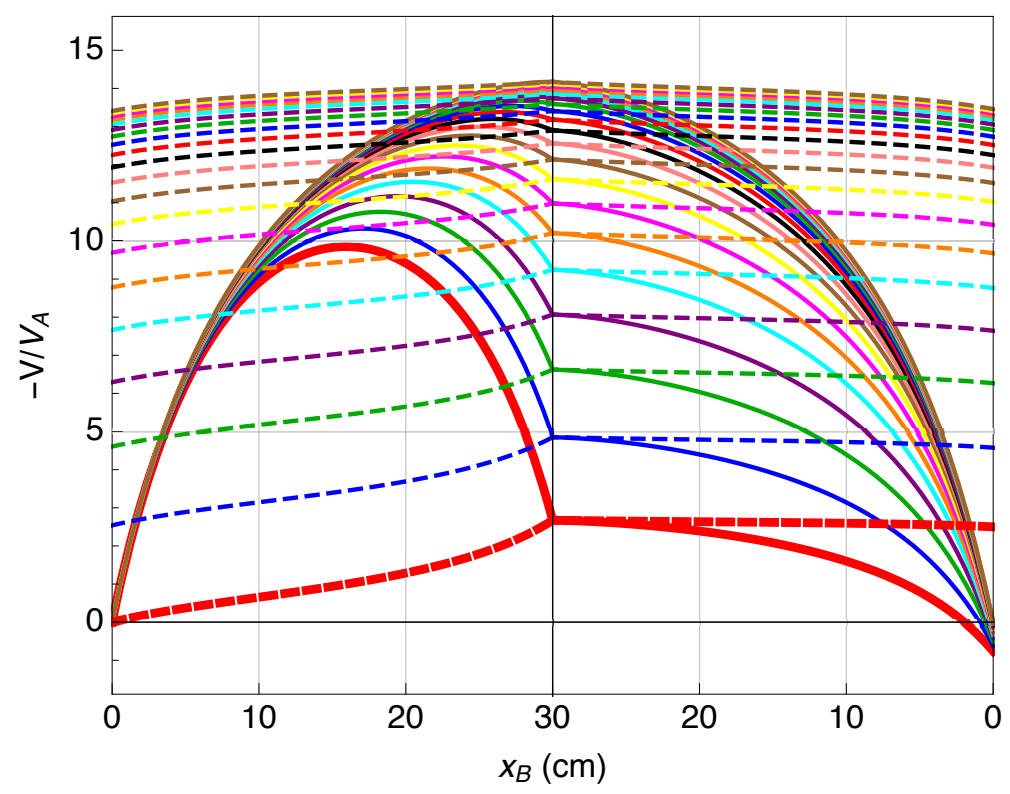

Figure 2. Voltages on plates B (solid lines) and C (dashed lines) in the simple model using the ideal parallel plate capacitance formula, over several charging cycles, starting with zero initial charge on plate $\mathrm{C}$. A cycle starts on the left $\left(x_{B}=0\right)$ with plate $\mathrm{B}$ grounded, proceeds to the right with $\mathrm{B}-\mathrm{C}$ contact at $x_{B}=30 \mathrm{~cm}$, and continues to the right as plate $\mathrm{B}$ moves away from $\mathrm{C}$ after contact, returning to $x_{B}=0$ with a non-zero voltage prior to grounding. The thicker lines correspond to the first cycle.

dimensions of the plate and properties of the fluid, and it is proportional to the velocity. Hence the rate of energy dissipation depends on $v^{2}$. For the more likely case of turbulent flow the power loss is dependent on $v^{3}$,

$$
P_{D}=F_{D} v=c_{D} A \rho v^{3} / 2
$$

where $A$ is the plate area, $\rho$ is the fluid density and $c_{D}$ is the drag coefficient that depends upon fluid properties and object shape. The case of a Cavallo multiplier operating in superfluid helium is discussed below in Sec. 4.

\subsection{Parallel-Plate Capacitor Approximation}

For further insight into the physics of Cavallo's machine, we model the mutual capacitances between the plates with the ideal parallel plate capacitor formula,

$$
C_{i j}=\frac{\epsilon_{0} A}{\left|x_{i}-x_{j}\right|}
$$

Results of applying the above analysis to this model are shown in figures 2 through 4 for the following parameters: diameter of plates $d=40 \mathrm{~cm}$, initial B-C distance (stroke length of the moving plate B) $L=30 \mathrm{~cm}$, capacitance of the target electrode to ground $C_{\mathrm{CG}}=70 \mathrm{pF}$, parasitic capacitance of plate $\mathrm{B}$ to ground $C_{\mathrm{BG}}=8 \mathrm{pF}$ (constant value; in a real system this would likely be lower when $\mathrm{B}$ is near $\mathrm{C}$ ).

Plate B obtains a high voltage on its way to $\mathrm{C}$ as its capacitance with other electrodes decreases (figure 2). In a real system, the ground shell needs to be sufficiently far away from B to prevent 


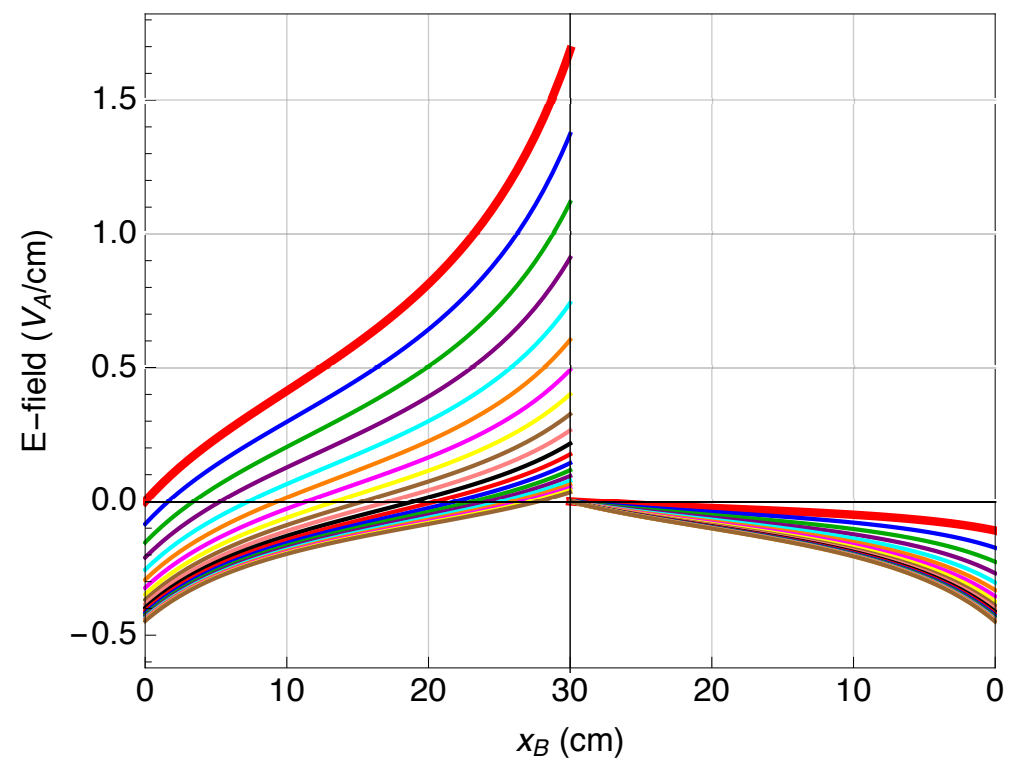

Figure 3. Electric field between plates B and C in the same model as in figure 2, with the same horizontal axis. The highest field occurs in the first cycle (plotted as a thicker line), and falls off in subsequent cycles.

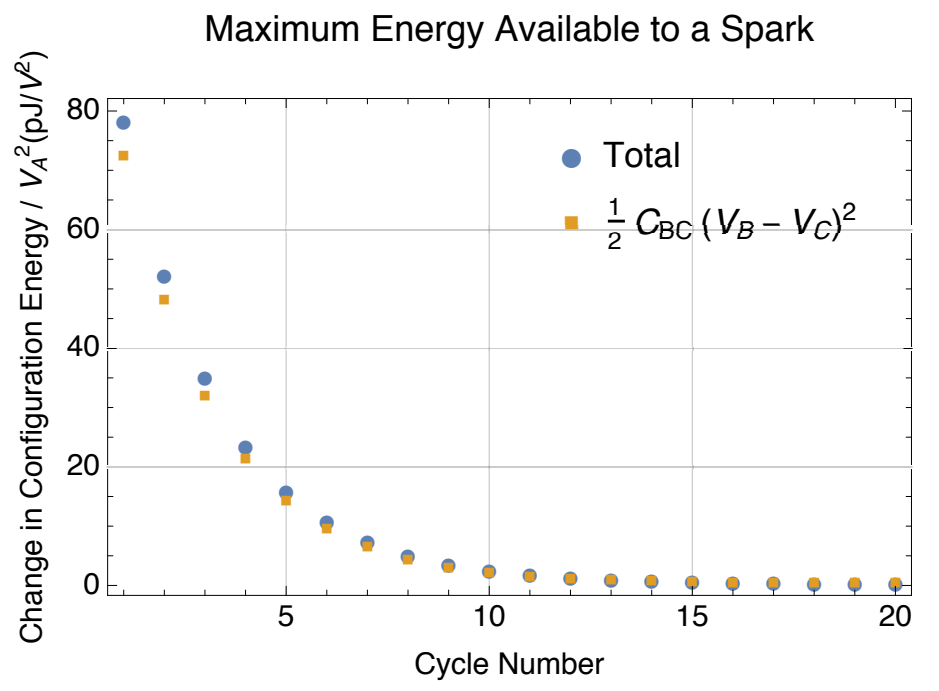

Figure 4. Energy available to a spark in the simple parallel-plate capacitor model, based on change in the configuration energy (eq. 2.18) at $0.5 \mathrm{~cm}$ separation between plates B and C, if short-circuit suddenly develops between these plates. The blue points are the total change in energy, and the orange points are $C_{B C}\left(V_{B}-V_{C}\right)^{2} / 2$ evaluated at the same $0.5 \mathrm{~cm}$ separation. 

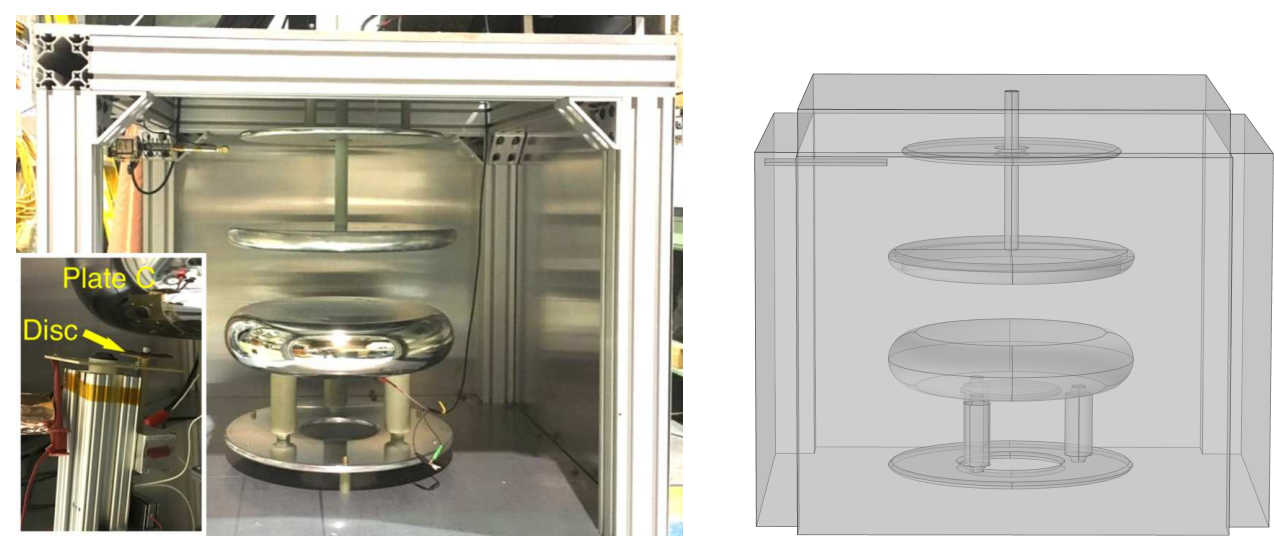

Figure 5. Left: photograph of the demonstration apparatus, with two of the grounded sides removed. Here, plate B is about halfway along its path to plate $\mathrm{C}$. The vibrating disc, non-contact electric field probe, not present in the main photograph but shown in the inset photograph, was positioned below and at the outer edge of plate C. Right: COMSOL [3] geometry used to compute mutual capacitances between the electrodes in the demonstration apparatus.

electrostatic discharge due to too strong of an electric field. As plate B nears $\mathrm{C}$, the voltages approach the same value before the plates contact. In this idealized model, the electric field between B and $\mathrm{C}$ steadily increases toward a finite value (figure 3). In practice, a surface feature or edge effect would create a localized high field and eventually a spark. A spark is modeled as a short circuit between $\mathrm{B}$ and $\mathrm{C}\left(V_{B}=V_{C}\right.$, and $Q_{B}+Q_{C}$ conserved). The maximum energy available to a spark, the change in total electrostatic potential energy (eq. 2.18), is plotted in figure 4 assuming the spark occurs at 5-mm B-C gap in each cycle. At this gap, the B-C capacitor accounts for most of the energy available to a spark, with the rest attributable to parasitic capacitances.

The highest field between $\mathrm{B}$ and $\mathrm{C}$ is on the first cycle, when $\mathrm{C}$ is initially uncharged. The highest spark energy is correspondingly on the first cycle. The charging voltage $V_{A}$ could be reduced for the first few cycles, allowing $\mathrm{B}$ and $\mathrm{C}$ to approach closer before the electric field exceeds some breakdown condition. The net energy deposited into sparks could thus be reduced. There is also a spark upon return of plate $\mathrm{B}$ to its position next to A, when it makes contact with the ground pin. In this case, $V_{A}$ could be adjusted (reduced) before $\mathrm{B}$ contacts ground to suppress the spark, and subsequently reset to its nominal value to more gently induce charge onto B.

\section{Demonstration apparatus}

We constructed an apparatus to test quantitative understanding of the voltage development on plate $\mathrm{C}$. The apparatus geometry is similar to that shown in figure 1, with electrode diameters of approximately $40 \mathrm{~cm}$ and stroke length $29 \mathrm{~cm}$. The Plate C electrode is supported by $\mathrm{G} 10$ insulators on a standoff ring, which was connected to common ground in these studies. The moving electrode, plate B, is supported by a solid G10 rod which passes through the top shelf to a linear actuator. Before moving away from its upper position next to plate A, plate B is electrically isolated from ground by retracting a solenoid-actuated grounding pin. A photograph of the apparatus is shown in figure 5, along with the apparatus geometry representation in the 


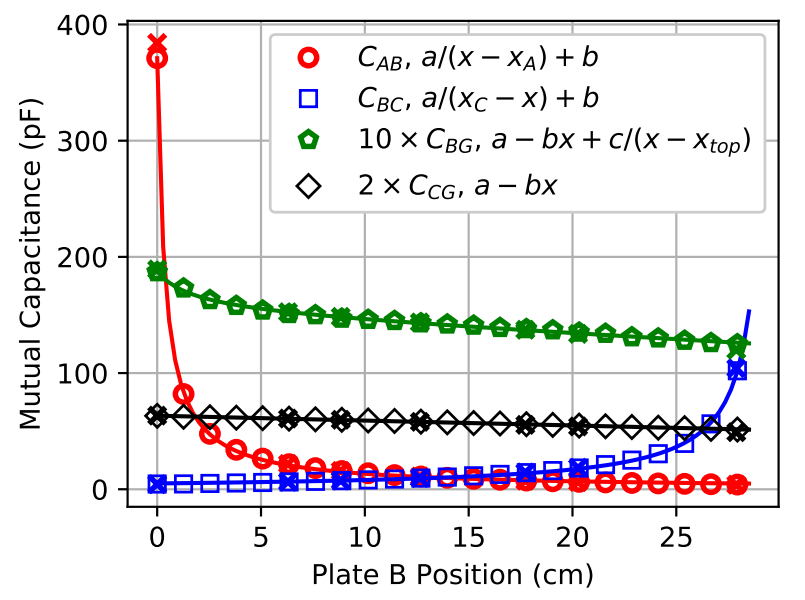

Figure 6. Mutual capacitances between electrodes in the demonstration apparatus, measured and calculated with COMSOL [3] (exes). Fits to the measured values by functions listed in the legend are shown, where $x$ is the horizontal axis and all other parameters are fitted. The data and fits for $C_{B G}$ and $C_{C G}$ are scaled for clarity in the plot. An additional grounded plate underneath Plate $\mathrm{C}$ was added later, which increased $C_{C G}$ by a constant $\approx 100 \mathrm{pF}$.

finite element software COMSOL [3], which was used to check measured capacitance values. Not present in the photograph and COMSOL model is an additional grounded electrode immediately underneath Plate $\mathrm{C}$ to increase load capacitance.

The mutual capacitances between pairs of electrodes in the system (including ground) were measured as a function of plate B position using a handheld capacitance meter (Agilent U1733C). A pair of coaxial cables served as measurement leads, with the outer conductors connected to the guard input on the meter. These cables were passed through the top deck of the apparatus, and electrodes $i$ and $j$ were connected to the meter inputs via the coaxial cables center conductors to measure $C_{i j}$. All electrodes in the system other than $i$ and $j$ were also connected to the guard input of the meter, and all grounded sides of the apparatus were in place during measurements. This arrangement was effective in removing parasitic capacitances, ensuring the measured value was the mutual capacitance $C_{i j}$. Results of these measurements are shown in figure 6 along with fits to empirical functions. The $C_{i j}$ calculated with finite element software are also plotted, showing good agreement with the measurements.

At the relatively small plate charges in the demo apparatus for reasonable applied voltages on Plate A, leakage current must be minimized. We employed a non-contact method to measure the voltage by measuring the current amplitude from a small $\left(\approx 8 \mathrm{~cm}^{2}\right)$ vibrating disc in the fringe field of Plate $\mathrm{C}$. The disc was positioned below Plate $\mathrm{C}$ near the outer edge (see figure 5 inset), where the influence of field lines from other non-grounded conductors is expected to be negligible. The current from the disc was amplified by a high-gain current-to-voltage preamplifier (Stanford Research SR556), which maintains the disc at virtual ground potential, followed by a lock-in amplifier (Stanford Research SR830). The reference oscillator from the lock-in amplifier was input into a high voltage operational amplifier (TI OPA548) driving a mechanically-amplified piezoactuator (Thorlabs PK2FVF1), with parameters set to move the disc with $\approx 40 \mu$ m amplitude, $40 \mathrm{~Hz}$ sinusoidal displacement. In this arrangement, the lock-in out-of-phase component was proportional 


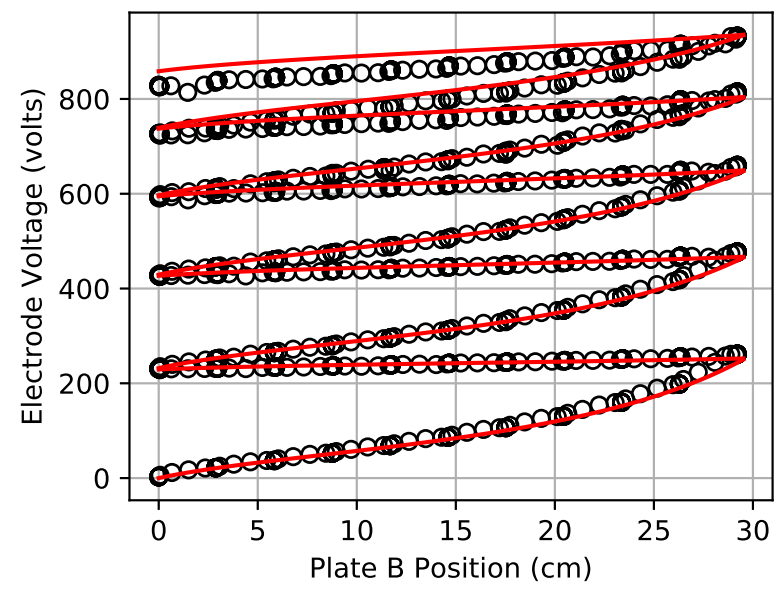

Figure 7. Voltage on the demonstration apparatus plate $\mathrm{C}$ during five charging cycles with $V_{A}=-100$ volts. Plotted are the measured voltages with the non-contact vibrating capacitor method (circles) and predicted voltages (line) based on the fits to measured mutual capacitances (figure 6).

to the voltage on Plate $\mathrm{C}$. The constant of proportionality between the lock-in signal and $V_{C}$ was calibrated by connecting an electrometer to Plate $\mathrm{C}$ and running a Cavallo charging cycle to sweep out $V_{C}$ voltages.

We note that a possible alternative non-contact electric field transducer for this measurement is the "field mill," which replaces the vibrating capacitor with a fixed capacitor that is alternately guarded from or exposed to the electric field by a rotating vane. That type of arrangement likely offers a much larger raw signal, but the vibrating capacitor method may be 1) more readily adapted to the strictly non-magnetic, sub-1 Kelvin cryogenic environment of the experiment discussed in Sec. 4; and 2) with suitable electrostatic design, less likely to cause sparking when operating in a high field region. However, with respect to point 1 , a field mill variant that may be suitable for cryogenic environments uses a micromachined vibrating aperature [4].

With the non-contact voltage measurement calibrated, the electrometer was disconnected, and several charging cycles were performed. Results are shown in figure 7, along with predicted voltages based on the fits to measured mutual capacitances (figure 6) and application of eqs. 2.10 and 2.11. Voltages in the first few cycles agree well with prediction, with some systematic disagreement especially in the last cycle. Possible causes of the disagreement include the cumulative effect of errors in capacitance measurements and leakage current through insulating standoffs. Although care was taken in the apparatus construction to avoid the triboelectric effect caused by the rod sliding against dissimilar material (such as a plastic bearing block in an earlier iteration), another possible source of systematic error is from charges stuck to the G10 rod holding Plate B, which would impact the charge initially induced on Plate B and that transferred to Plate C. At much higher operating voltages, this effect should be relatively less important. Leakage current through the insulators supporting plate $\mathrm{C}$ could in principle be accounted for by connecting the standoff ring to the input of a picoammeter (virtual ground), rather than directly to ground, and logging these data to enable a correction in the data analysis; however, this was not done in the present study. In any case, it appears the voltages in the demonstration apparatus can be reasonably well predicted 
from measured or calculated capacitances, validating an approach of using calculated capacitances to evaluate designs for a production apparatus.

\section{Cavallo's multiplier in a neutron EDM experiment}

As a specific example of incorporating a Cavallo multiplier into a physics experiment, we performed a high voltage design study for a large-scale, cryogenic experiment to search for the neutron electric dipole moment (nEDM) [5, 6]. The central detector is a large $\left(\sim 1 \mathrm{~m}^{3}\right)$ volume containing liquid helium at about 0.4 Kelvin. A capacitor is formed by a central high voltage electrode flanked on either side by measurement cells followed by ground electrodes. To take advantage of the excellent dielectric strength of liquid helium, a design goal is to charge this capacitor, with $C_{C G} \approx 100 \mathrm{pF}$, to about $650 \mathrm{kV}$, corresponding to a field inside the measurement cells of $75 \mathrm{kV} / \mathrm{cm}$.

Bringing $650 \mathrm{kV}$ into a volume filled with LHe at $0.4 \mathrm{~K}$ with a direct $\mathrm{HV}$ feed is a very challenging task. The HV feed line needs to be designed so that the heat brought in is within the allowed heat budget of a few tens of milliwatts, which requires proper thermal anchoring at $4 \mathrm{~K}$ and $77 \mathrm{~K}$. In addition, the feedthrough needs to be superfluid tight and, for the nEDM experiment, strictly non-magnetic. The leakage current flowing across the surface of the insulator must be minimized to avoid heating. Note that a leakage current of $15 \mathrm{nA}$ flowing across the surface of a $650 \mathrm{kV}$ HV feedthrough insulator would cause a heating of $10 \mathrm{~mW}$. Bringing in $50 \mathrm{kV}$ is relatively easy, and compact (few centimeter scale) commercial feedthroughs are available with $\sim 1 \mathrm{nA}$ leakage current [7, 8]; therefore, we set $V_{A}=50 \mathrm{kV}$. A stable field of $100 \mathrm{kV} / \mathrm{cm}$ in liquid helium with large electrodes has been demonstrated [7], so we set this as the maximum allowed field in this design study.

The starting point is a Cavallo multiplier with geometry similar to figure 1, with a conducting rod between plate $\mathrm{C}$ and the central high voltage electrode in the measurement cell structure. The maximum achievable voltage gain $G^{\max }$ (eq. 2.7), obtained asymptotically with increasing number of charging cycles, is a useful guide for choosing an initial electrode geometry. In a practical application with a reasonable number of cycles, we could charge the system until for example $\approx 90 \%$ of the maximum gain is reached; thus, our goal is $G^{\max }=13 / 0.9=14.4$. Consistent with a maximum electric field of $100 \mathrm{kV} / \mathrm{cm}$, the initial A-B gap is set to $5 \mathrm{~mm}$, with appropriate Rogowski profiles $[9,10]$ for plates A and B to avoid high electric field at the plate edges. Increasing $C_{A B}^{a}$, without increasing the electric field strength, requires increasing the plate area. The other capacitances in eq. 2.7 should be minimized for the highest voltage gain. Increasing the plate B stroke length $\left(x_{C}\right)$ decreases $C_{A B}^{c}$ and $C_{B C}^{a}$. The remaining significant capacitance affecting the gain is $C_{B G}^{c}$, the parasitic capacitance of $\mathrm{B}$ to ground when next to plate $\mathrm{C}$. This can be reduced by adding lobes to plate $\mathrm{C}$ to partially envelop $\mathrm{B}$, partially shielding it from ground.

We performed a finite-element study using COMSOL [3] to find a nominal geometry appropriate for the nEDM apparatus. The purpose was to evaluate mutual capacitances between conducting bodies and look for electric field hot spots, and several iterations were performed. Given the capacitances from COMSOL evaluated when B is at its initial (next to A) and final (touching C) positions, the formulae from the analysis in section 2 can be applied. This is shown in figure 8 , the voltage on the $\mathrm{C}$ electrode versus charging cycle number. The electric field distribution when $\mathrm{C}$ is charged 

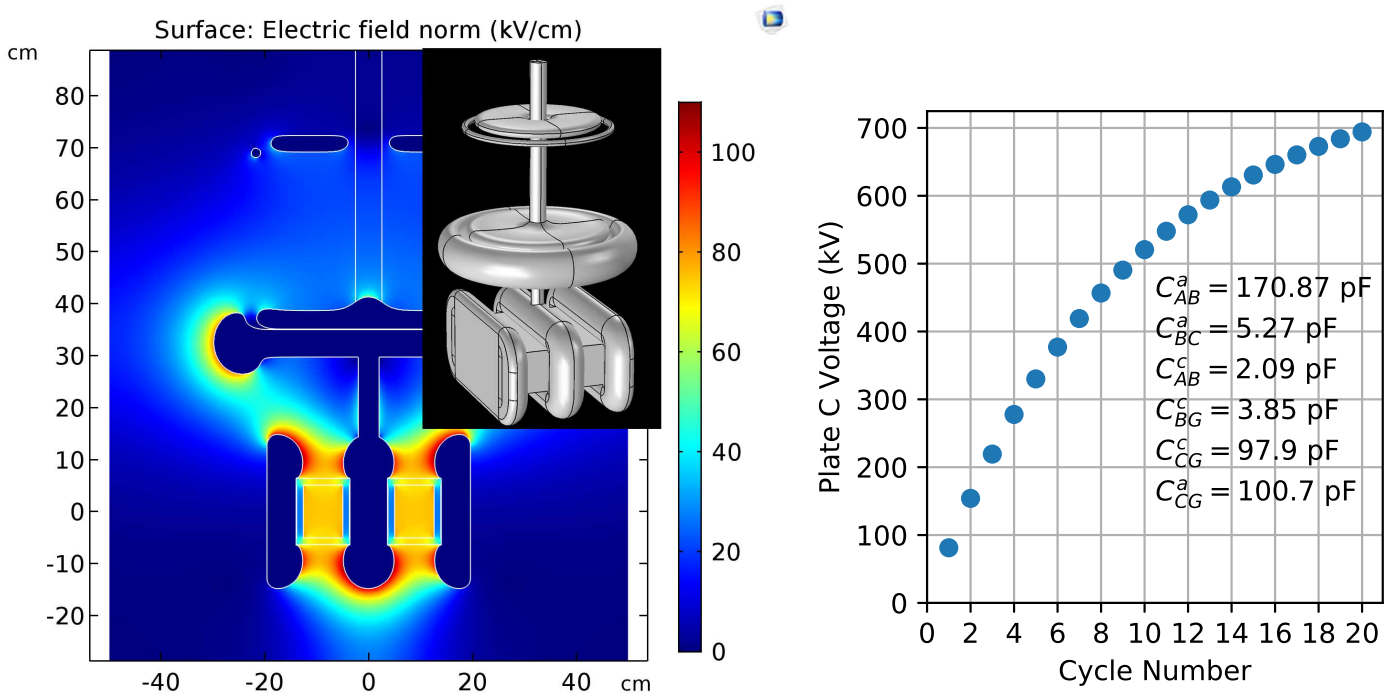

Figure 8. Full 3D COMSOL [3] simulation of the nEDM central volume including a ground return at $50 \mathrm{~cm}$ radius. Left: electric field strength at the midplane when $\mathrm{C}$ is fully charged to $650 \mathrm{kV}$ and plate $\mathrm{B}$ remains in contact with $\mathrm{C}$ (the large electrode in the middle). The highest field around plate $\mathrm{C}$ is about $80 \mathrm{kV} / \mathrm{cm}$. Right: calculated $V_{C}$ vs. number of charge cycles completed, using the computed mutual capacitances and application of eqs. 2.10 and 2.11 .

to $650 \mathrm{kV}$ is shown in figure 8 . The $\mathrm{A}$ and $\mathrm{B}$ electrodes have diameter $40 \mathrm{~cm}$, and the part of the $\mathrm{C}$ electrode in the middle of the figure 8 has lobes around its perimeter to reduce $C_{B G}^{c}$.

For this design to be feasible, the heat load must be small, less than about $10 \mathrm{~mW}$ averaged over a $\sim 100$-second total charging period. The heat energy input due to charge flow across resistive electrodes appears to be negligible: $W_{q} \sim \rho_{S} Q_{B}^{2} / \Delta t \sim 1 \mathrm{~nJ}$ per cycle, with plate resistivity $\rho_{S} \sim 1 \mathrm{k} \Omega / \square$, initial charge $Q_{B} \sim 1 \mu \mathrm{C}$, and characteristic charge movement time $\Delta t \sim 1 \mathrm{~s}$. For the energy deposited by sparks, the parallel plate toy model in section 2.3 is relevant, which shows a spark energy (figure 4) of at most $W_{s} \sim\left(80 \mathrm{pJ} / \mathrm{V}^{2}\right) \times(50 \mathrm{kV})^{2}=0.2 \mathrm{~J}$ in the first cycle and falling off quickly in subsequent cycles. If needed, $W_{s}$ can be reduced by lowering $V_{A}$ for the first few cycles, when the field between plates $\mathrm{B}$ and $\mathrm{C}$ would otherwise be much higher than in later cycles (see figure 3), possibly allowing $\mathrm{B}$ to approach closer to $\mathrm{C}$ before sparking. Constructing the electrodes with designated contact areas made of high dielectric strength, bulk-resistive material could decrease the instantaneous power of the sparks by slowing charge transfer between electrodes, possibly avoiding electrode surface damage but not reducing the total heat production due to charge transfer.

The motion of the B electrode in superfluid helium at $0.4 \mathrm{~K}$ can create heat by two mechanisms. Firstly, a drag force arises from the motion of an object through the thermal phonon excitations existing in the liquid, the classical analogue of dissipation in laminar flow. And secondly, when the velocity of the object reaches a critical value, quantum turbulence, an entanglement of quantized vortices which has no classical correspondence, is generated. Measurements have been made of these phenomena at low temperature by the motion of oscillating spheres, wires, grids and tuning forks [11] but at sizes of millimeters or smaller. Results obtained at these very small scales must be extrapolated by orders of magnitude to obtain estimates for heat produced by the motion of the 
$40 \mathrm{~cm}$ diameter movable electrode.

The experiments by Niemetz and Schoepe [12] on a $124 \mu \mathrm{m}$ oscillating sphere are perhaps the most directly related to the case of a moving plate. From their data for the phonon drag force at $0.3 \mathrm{~K}$, which has a magnitude of piconewton-scale, the estimated heat produced by the $40-\mathrm{cm}$ diameter electrode with a speed of $3 \mathrm{~cm} / \mathrm{s}$ in helium at $0.4 \mathrm{~K}$ is the order of $20 \mu \mathrm{W}$ and is no concern. However, the velocity of $3 \mathrm{~cm}$ is well above the critical velocity for the generation of quantum turbulence by the electrode [13]. The drag force, $F_{D}$, from quantum turbulence is found to vary with velocity, $v$, approximately as $F_{D} \approx \gamma v^{2}$, where the coefficient, $\gamma$, when expressed in the form of the drag coefficient for classical turbulence, eq. 2.20, is $\gamma=c_{D} \rho A / 2$. The fact that the drag from quantum turbulence can be characterized by the same general expressions as for classical hydrodynamics provides some confidence that the estimated heat production by the electrode, obtained by extrapolating measurements made on objects 3 orders of magnitude smaller in size, can be reliable. Upon taking in into account the difference in size and shape of the electrode compared to the oscillating sphere used by Niemetz [12], the estimated heat input due to the production of quantum turbulence by the electrode moving at $3 \mathrm{~cm} / \mathrm{s}$ is a factor of $10^{7}$ larger than the sphere with the same rms velocity, but nonetheless is somewhat less than $1 \mathrm{~mW}$, an acceptable rate for the nEDM apparatus.

\section{Discussion and Conclusion}

A Cavallo multiplier may be a good option for experiments that require a very high voltage but very low current, such as electric dipole moment experiments. Any significant leakage current can be addressed by periodically transferring additional charge to the load capacitor, with the charging voltage $V_{A}$ adjusted to stabilize the load voltage $V_{C}$. If the experiment is performed as a series of measurement cycles that include a preparation period (e.g., loading particles into a measurement cell) and a separate measurement period, the charging cycle can be executed outside the measurement time interval. The voltage during the measurement period will slowly decrease in magnitude, depending on the leakage current, but will have zero ripple, a possible advantage over other methods of high voltage production in sensitive experiments.

The Cavallo multiplier is a simple device amenable to good electrostatic design, and it can be made compatible with a cryogenic environment. In the case of a liquid helium filled vessel, the device makes good use of the high dielectric strength of the medium [7]. An initial design study indicates that the Cavallo multiplier is feasible for a large-scale, cryogenic experiment to measure the neutron electric dipole moment. Another possible application is in time projection chambers (TPCs) [14] operated in liquid nobles such as argon and xenon [15, 16]. An electric field of 500$1000 \mathrm{~V} / \mathrm{cm}$ is needed to drift electrons generated by passage of charged particles to the detector grid. Currently, the length of the drift region is limited by the performance of the high voltage feedthrough $[17,18]$. Liquid nobles, which include helium, have high dielectric strengths, and similar electrostatic design constraints apply as in the Cavallo multiplier for the nEDM experiment (Sec. 4).

A TPC requires a constant current to flow from the anode to the cathode through the field cage resistor chain to maintain a uniform electric field in the drift region. The amount of current flowing in the resistor chain must be much greater than the expected ionization current in the drift region. 
Depending on the current requirement and other specifications like voltage stability, a Cavallo multiplier periodically executing charging cycles to top off the load capacitance $C_{C G}$ may not be sufficient. One could imagine arranging several Cavallo multipliers in parallel to increase the supply current, with the charging cycles run out of phase to even out the voltage on the shared load capacitor. However, an arrangement similar to the high voltage generator of Pelletron accelerators [2], which is essentially a continuous version of a Cavallo multiplier in which plate B is divided into many small segments and arranged to operate sequentially, may more suited for this application.

\section{Acknowledgements}

This work was supported by the U.S. Department of Energy, Office of Science, Office of Nuclear Physics contract number DE-AC52-06NA25396 under Field Work Proposal 2019LANLEEDM, and the National Science Foundation (1506459).

\section{References}

[1] T. Cavallo, A Complete Treatise on Electricity, Vol. III, 4th Edition (C. Dilly, London, 1795), 98-107.

[2] R.G. Herb, "Pelletron accelerators for very high voltage," Nucl. Instrum. Meth. 122, 267-276 (1974).

[3] COMSOL, Inc., COMSOL Multiphysics Reference Manual, version 5.3, http://www . comsol .com.

[4] M.N. Horenstein and P.R. Stone, "A micro-aperature electrostatic field mill based on MEMS technology," J. Electrostatics, 51-52, 515-521 (2001).

[5] R. Golub and S.K. Lamoreaux, "Neutron electric-dipole moment, ultracold neutrons and polarized ${ }^{3}$ He," Phys. Rep. 237 (1), (1994) 1-62.

[6] T.M. Ito, "Plans for a neutron EDM experiment at SNS," J. Phys.: Conf. Ser. 69, 012037 (2007).

[7] T.M. Ito et al., "An apparatus for studying electrical breakdown in liquid helium at $0.4 \mathrm{~K}$ and testing electrode materials for the neutron electric dipole moment experiment at the Spallation Neutron Source,” Rev. Sci. Instrum. 87, 045113 (2016).

[8] Off-the-shelf commercial feedthroughs may not be suitable for the nEDM apparatus due to strict limits on magnetic materials, but a similar, custom feedthrough using only non-magnetic materials appears feasible.

[9] W. Rogowski, "Die elektrische Festigkeit am Rande des Plattenkondensators. Ein Beitrag zur Theorie der Funkenstrecken um Durchfülhrungen," Archiv für Electrotechnik 12 (1), 1-15 (1923).

[10] J.D. Cobine, Gaseous Conductors: Theory and Engineering Applications (Dover, 1958, reprint of McGraw-Hill, 1941), 177-181.

[11] W.F. Vinen, “Quantum turbulence: achievements and challenges," J. Low Temp. Phys. 161, 419-444 (2010).

[12] M. Niemetz and W. Schoepe, "Stability of laminar and turbulent flow of superfluid ${ }^{4} \mathrm{He}$ at $\mathrm{mK}$ temperatures around an oscillating microsphere," J. Low Temp. Phys. 135, 447-469 (2004).

[13] N.B. Kopnin, "Vortex instability and the onset of superfluid turbulence," Phys. Rev. Lett. 92, 135301 (2004).

[14] J.N. Marx and D.R. Nygren, “The Time Projection Chamber," Physics Today 31 (10), 46-53 (1978). 
[15] A. Rubbia, "Future liquid Argon detectors," Nucl. Phys. B (Proc. Suppl.) 235-236 190-197 (2013).

[16] E. Aprile et al., "The XENON100 dark matter experiment," Astroparticle Physics 35, 573-590 (2012).

[17] B. Rebel et al., "High voltage in noble liquids for high energy physics," JINST 9 (08), T08004 (2014).

[18] C. Cantini et al., "First test of a high voltage feedthrough for liquid Argon TPCs connected to a 300 kV power supply," JINST 12, P03021 (2017). 